\title{
Study of the Growth of Capped ZnO Nanocrystals: A Route to Rational Synthesis
}

\author{
Ranjani Viswanatha and D. D. Sarma*[a]
}

\begin{abstract}
We report the study of complex and unexpected dependencies of nanocrystal size as well as nanocrystalsize distribution on various reaction parameters in the synthesis of $\mathrm{ZnO}$ nanocrystals using poly(vinyl pyrollidone) (PVP) as a capping agent. This method establishes a qualitatively different growth mechanism to the anticipated Ostwald ripening behavior. The study
\end{abstract}

of size-distribution kinetics and an understanding of the observed non-monotonic behaviors provides a route to rational synthesis. We used a simple, but

Keywords: crystal growth - crystalsize distribution - nanotechnology • semiconductors • time-resolved spectroscopy accurate, approach to estimate the sizedistribution function of nanocrystals from the UV-absorption spectrum. Our results demonstrate the accuracy and generality of this approach, and we also illustrate its application to various semiconducting nanocrystals, such as $\mathrm{ZnO}, \mathrm{ZnS}$, and $\mathrm{CdSe}$, over a wide size range $(1.8-5.3 \mathrm{~nm})$.

\section{Introduction}

Semiconducting nanocrystals, whose optical and electronic properties can be continuously tuned by varying the size of the particles, are promising candidates for future applications. ${ }^{[1]}$ To tailor these properties of nanocrystals for specific applications, it is essential to be able to control not only the average size, but also the size distribution. Chemical syntheses provide opportunities to control both the size and the size distribution by varying the concentration of the reactants, capping agents, and the temperature.

$\mathrm{ZnO}$ (bulk bandgap of $3.3 \mathrm{eV}$ ) is a useful material for a wide range of applications, such as solar cells, luminescent devices, and chemical sensors. ${ }^{[1]}$ It is one of the few oxides with a reasonably wide and experimentally accessible size range $(<7 \mathrm{~nm})$ that facilitates a substantial tunability of the bandgap, primarily because of the large excitonic diameter $(\sim 5.6 \mathrm{~nm})$. We chose this specific system based on recent interest in synthesizing high-quality $\mathrm{ZnO}$ nanocrystals of various sizes, including transition-metal-doped $\mathrm{ZnO}$ nanocrystals. ${ }^{[2-6]}$ Although the growth mechanism of $\mathrm{ZnO}$ in the ab-

[a] R. Viswanatha, Prof. D. D. Sarma

Solid State and Structural Chemistry Unit

Indian Institute of Science, Bangalore-560012 (India)

Fax: (+91) 80-2360-1310

E-mail: sarma@sscu.iisc.ernet.in

Supporting information for this article is available on the WWW under http://www.chemeurj.org/ or from the author: UV-absorption curves and details of the effect of water on growth. sence of any capping agent is reported to be that of Ostwald ripening, ${ }^{[7]}$ the practical synthesis of $\mathrm{ZnO}$ nanocrystals, as for almost all nanocrystals, is invariably carried out in the presence of a capping agent to stabilize the desired size for a given application. However, very little is known about the growth process in such a complex reaction. To optimally control parameters in such a reaction, it is necessary to understand the influence that each parameter has on the average size and the size distribution. Traditionally, transmission electron microscopy (TEM) has been used to measure these two quantities. Although the average size can be estimated from other experimental techniques, such as X-ray diffraction and optical absorption spectroscopy, the size distribution of an assembly of nanocrystals has so far been determined solely from time-consuming TEM studies. Moreover, it is impossible to follow both the size and the size distribution of a fast-growing particle during synthesis by using this technique. Hence, it is necessary to devise other methods to follow the size distribution of the particles during the reaction in real time.

The sharpness of the UV-absorption edge and the presence of an excitonic peak have long been used to qualitatively establish the relative degree of monodispersity in nanocrystal samples; a relatively sharp edge with a well-defined excitonic peak suggesting a narrow size dispersion, and a broad absorption edge denoting a large size dispersity. It is indeed evident that the spread of the absorption edge indicates the size distribution in view of the sensitive dependence of the bandgap, and consequently of the absorp- 
tion edge, on size. Here, we study the effect of $[\mathrm{OH}]^{-}$and poly(vinyl pyrollidone) (PVP) concentrations on the synthesis of capped $\mathrm{ZnO}$ nanocrystals as a function of time. We show that the UV-visible absorption spectra can be analyzed to obtain quantitatively accurate estimates of size dispersions in a variety of systems. In addition, we use this method to establish several unexpected, non-monotonic dependencies of the size and the size distribution of the $\mathrm{ZnO}$ nanocrystals on the reaction conditions.

\section{Results and Discussion}

The authors of reference [4] reported a UV-absorption spectrum and a transmission electron micrograph of a $\mathrm{ZnO}$ nanocrystal sample, providing an ideal starting point for the application of this method. We estimated the size-distribution histogram for this sample (Figure 1a) by using the re-

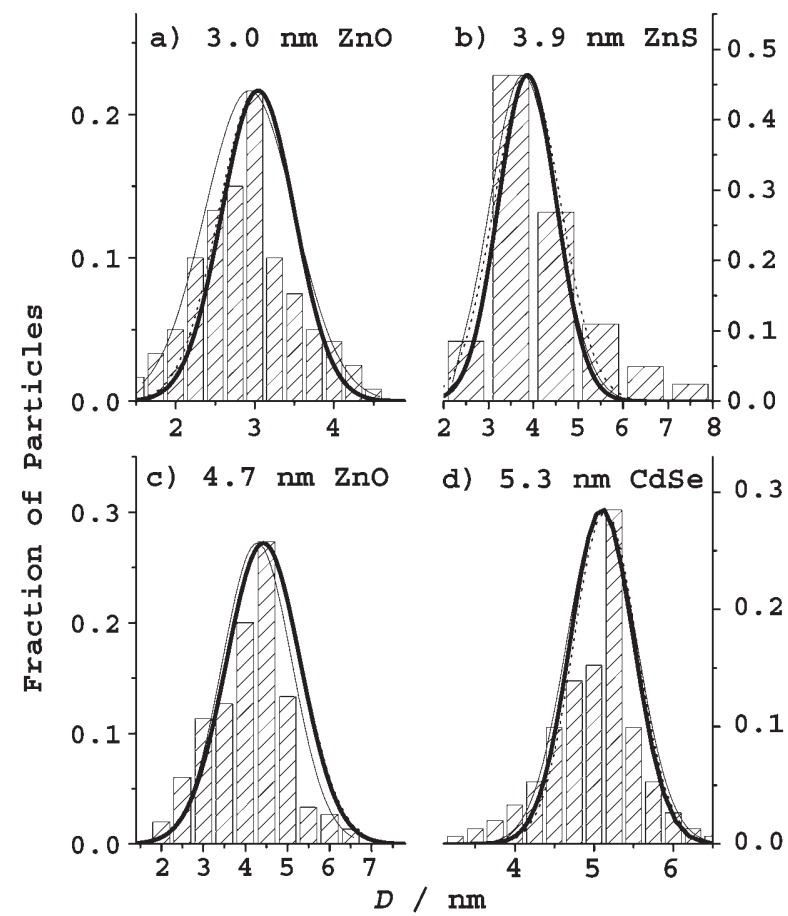

Figure 1. Comparison of the size distribution obtained from fitting the UV-absorption curve (thick solid line) with the TEM histogram (column plot) of (a) $3.0 \mathrm{~nm}$ particles of $\mathrm{ZnO}$, (b) $3.9 \mathrm{~nm}$ particles of $\mathrm{ZnS}$, (c) $4.7 \mathrm{~nm}$ particles of $\mathrm{ZnO}$, and (d) $5.3 \mathrm{~nm}$ particles of CdSe. The corresponding fits by broadening an infinitely sharp function and Gaussian fit to the TEM histogram are shown as dotted lines and thin solid lines, respectively.

ported micrograph. The size distribution obtained from the TEM was fitted to a Gaussian function, shown as a thin solid line in Figure 1a, to yield estimates for the average size, $D_{\mathrm{av}}^{\mathrm{TEM}}=2.95 \mathrm{~nm}$, and the size dispersion in terms of the full width at half maximum (FWHM), $\Delta D^{\mathrm{TEM}}=1.13 \mathrm{~nm}$. The size-distribution function obtained by analyzing the reported UV-absorption spectrum gives the optimized values of $D_{\mathrm{av}}^{\mathrm{UV}}(3.09 \mathrm{~nm})$ and $\Delta D^{\mathrm{UV}}(1.11 \mathrm{~nm})$. The resulting distribution function, shown by a thick solid line in Figure 1a, is very similar to that obtained from TEM analysis (thin solid line); specifically the differences in the $D_{\text {av }}$ and $\Delta D$ values obtained from the two techniques are less than $5 \%$ and $2 \%$, respectively. To ensure that this good agreement is not coincidental, we conducted similar analyses for another $\mathrm{ZnO}$ nanocrystal sample with a much larger average size, as well as for ZnS nanocrystals and CdSe nanocrystals whose UVabsorption spectra and TEM results have already been reported. $^{[4,8,9]}$ Figure $1 \mathrm{~b}-\mathrm{d}$ shows comparisons between sizedistribution functions obtained by analyzing UV-absorption spectra (thick solid lines) and the corresponding TEM results (thin solid lines), once again showing good overall agreement in each case. Quantitatively, $D_{\text {av }}^{\mathrm{TEM}}=4.2 \mathrm{~nm}$ and $D_{\mathrm{av}}^{\mathrm{UV}}=4.35 \mathrm{~nm}$, with $\Delta D^{\mathrm{TEM}}=1.98 \mathrm{~nm}$ and $\Delta D^{\mathrm{UV}}=2.02 \mathrm{~nm}$ for the $\mathrm{ZnO}$ sample; $D_{\mathrm{av}}^{\mathrm{TEM}}=3.8 \mathrm{~nm}$ and $D_{\mathrm{av}}^{\mathrm{UV}}=3.95 \mathrm{~nm}$, with $\Delta D^{\mathrm{TEM}}=1.7 \mathrm{~nm}$ and $\Delta D^{\mathrm{UV}}=1.55 \mathrm{~nm}$ for the $\mathrm{ZnS}$ sample; and $D_{\mathrm{av}}^{\mathrm{TEM}}=5.10 \mathrm{~nm}$ and $D_{\mathrm{av}}^{\mathrm{UV}}=5.13 \mathrm{~nm}$, with $\Delta D^{\mathrm{TEM}}=$ $1.05 \mathrm{~nm}$ and $\Delta D^{\mathrm{UV}}=0.95 \mathrm{~nm}$ for the CdSe sample. The largest discrepancies between estimates from UV-absorption and TEM data are less than $5 \%$ and $10 \%$ for $D_{\text {av }}$ and $\Delta D$, respectively. Notably, the use of the effective mass approximation (EMA) leads to a drastic overestimation of $D_{\mathrm{av}}^{\mathrm{UV}}$ and an underestimation of $\Delta D^{\mathrm{UV}}$, due to the well-known overestimation of the bandgap variation within the EMA. This is in contrast to the more accurate results for the bandgap shift with size obtained here. ${ }^{[4,10]}$

As discussed above, the experimental UV-absorption spectra of several different systems provide reliable information about the average size and the size distribution of these systems. This prompted us to explore whether this is universally true for any such system, which could lead to an easyto-use methodology for estimating these two quantities. Because only limited experimental data is available so far for many of these interesting systems, we simulated the UV-absorption data for different size distributions of a large number of systems, namely $\mathrm{ZnO}, \mathrm{ZnS}, \mathrm{ZnSe}, \mathrm{CdS}$, CdSe, GaAs, InAs, and InP. Assuming average size and size-distribution width with a Gaussian-distribution function, the expected UV-absorption curve is easily simulated with the help of the known bandgap shifts ${ }^{[4,14,15]}$ with size, as described in the Experimental Section. A typical example of a simulated UV-absorption spectrum for the case of GaAs, for which very little experimental data is available so far, is shown in part I of Figure 2b, for an average size of $3.5 \mathrm{~nm}$ and a relative percentage distribution of $8.5 \%$. Differentiation of this absorption spectrum produces the curve shown in part II. The differentiated curve defines a peak energy $E_{0}$, and an energy width in terms of $E_{1}$ and $E_{2}$, forming the FWHM. The absorption energy $E_{0}$ is readily translated into the average size, $d_{\mathrm{av}}$, by using the simple formula reported previously. ${ }^{[4,14,15]}$ Similarly, $E_{1}$ and $E_{2}$ can also be translated to apparent sizes, for example, $d_{1}$ and $d_{2}$. This then defines the apparent relative percentage distribution, $\Delta d_{\text {app }}=$ $\left(d_{1}-d_{2}\right) / d_{\mathrm{av}} \times 100$. This apparent value is much larger than the actual value that was based on the originally assumed 


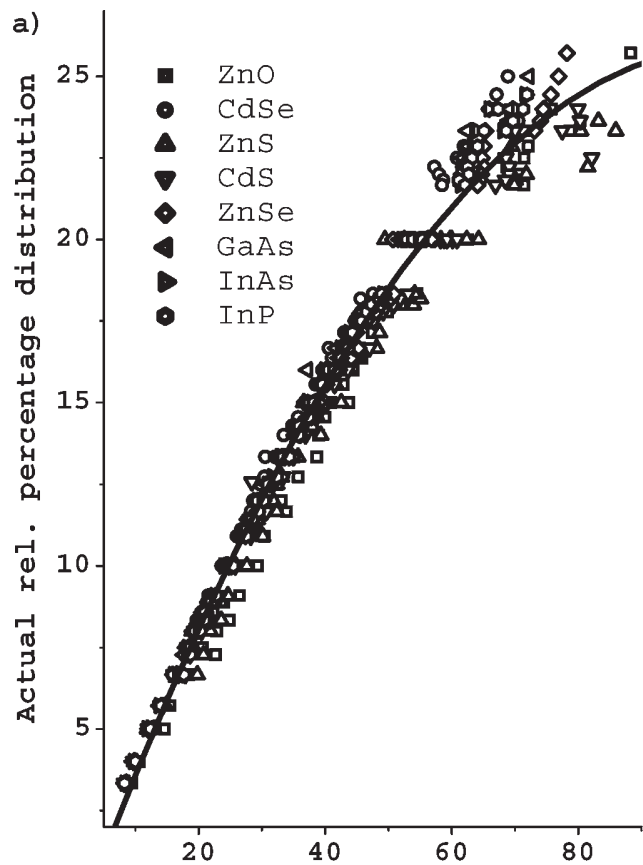

App. rel. percentage distribution

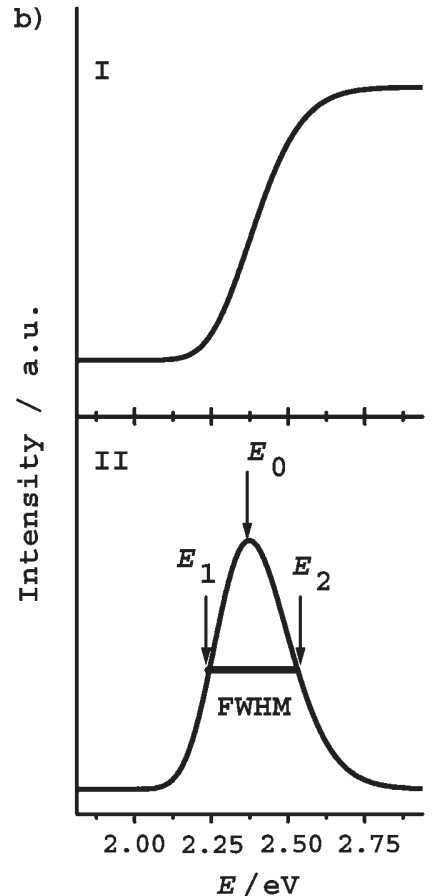

$E / \mathrm{eV}$

Figure 2. a) Correlation of the apparent relative percentage distribution obtained from UV-absorption curves with the actual relative percentage distribution for various II-VI and III-V systems. b) A typical example of simulated absorption data for $3.5 \mathrm{~nm}$ GaAs nanocrystals with an assumed relative percentage distribution of $8.5 \%$. Part II shows the derivative of the curve in part I, and the positions of $E_{0}, E_{1}$, and $E_{2}$.

distribution function. For example, in the illustrative example shown in Figure $2 \mathrm{~b}$, the $d_{\mathrm{av}}$ calculated from $E_{0}$ is $3.49 \mathrm{~nm}$, in very good agreement with the assumed value of $3.5 \mathrm{~nm}$. However, the apparent relative percentage distribution of $20.6 \%$ arising from $d_{1}=3.9 \mathrm{~nm}$ for $E_{1}=2.18 \mathrm{eV}$ and $d_{2}=3.17 \mathrm{~nm}$ for $E_{2}=2.41 \mathrm{eV}$ is about 2.5 times larger than the originally assumed value. Figure 2 a shows the plot of the actual relative percentage distribution as a function of apparent relative percentage distribution calculated on the basis of such simulated UV-absorption data. The plot summarizes our analyses of 720 cases of eight different compounds spanning the entire relevant size $(2-6 \mathrm{~nm})$ and relative percentage distribution (5-25\%) ranges. Interestingly, the plot reveals an almost universal behavior, as suggested by a simple quadratic fit (solid line) of the data points. This allows us to calibrate the apparent width from the analysis of the UV-absorption data and, subsequently, to obtain the true width. The fit describes the actual relative percentage distribution $\Delta D=A \times \Delta d_{\mathrm{app}}{ }^{2}+B \times \Delta d_{\mathrm{app}}+C$, in which $A, B$, and $C$ are equal to $-0.0025,0.524$, and -1.41 , respectively. By using the above example of GaAs, the derived apparent relative percentage distribution of $20.6 \%$ translates to an actual relative percentage distribution of $8.4 \%$, which is in remarkable agreement with the originally assumed relative percentage distribution of $8.5 \%$. The universality of this approach, attributable to the fact that the constants $A, B$, and $C$ are system and size independent, makes this approach particularly simple to use. Therefore, for any system of semiconducting nanocrystals, a simple differentiation of the UV- absorption spectrum directly yields the average size from the peak energy, and the apparent relative percentage distribution in conjunction with the relationship $\Delta D_{\text {actual }}=-0.0025 \times$ $\Delta d_{\text {app }}^{2}+0.524 \times \Delta d_{\text {app }}-1.41$ provides the estimate of the actual relative percentage distribution.

These results for different semiconductor nanocrystal systems, spanning a wide range of average sizes, establish the general validity of this approach. An additional advantage of this method stems from the insensitivity of the absorption spectra on surface and defect states, which, for example, influence the emission properties drastically. Therefore, a similar approach based on the emission spectra of nanocrystals ${ }^{[10]}$ will be limited only to perfectly passivated samples exhibiting bandedge emission, and would not be applicable to nanocrystals, such as $\mathrm{ZnO}$, that do not show a bandedge tuning. This method based on the absorption edge of the bulk, however, may be limited by the difference in the spectral shapes of the bulk and the nanocrystals, the latter generally exhibiting a sharper absorption edge than that of the bulk. To estimate the possible error from the use of the bulk spectrum, we also analyzed the results of the nanocrystals in the opposite limit, assuming that the absorption edge of an individual nanocrystal is infinitely sharp. In this case, the reference spectrum for the analysis, instead of being the experimental bulk spectrum, is represented by a step function, broadened by the instrumental resolution. The corresponding results of our analysis are plotted in Figure 1 as dotted lines; it can be seen that the difference between the results obtained under the assumptions of the two opposite, extreme limits is negligible, providing further credence to the present approach. However, it is important to note that the present approach does not allow us to analyze a bimodal distribution unless prior knowledge of the presence of such a distribution is available from independent measurements, such as TEM data. Nevertheless, the simplicity and very short application time of the absorption technique compared with the more conventional method of TEM to obtain the size distribution supports the use of this method in a time-resolved study of the effect of concentration of $\mathrm{NaOH}$ and $\mathrm{PVP}$ in the synthesis of $\mathrm{ZnO}$ nanocrystals.

The UV-absorption spectra of a typical reaction with a fixed $\mathrm{NaOH}$ concentration of $0.5 \mathrm{mmol}$ in $25 \mathrm{~mL}$ as a function of time is shown in the inset of Figure 3. There is a sys- 


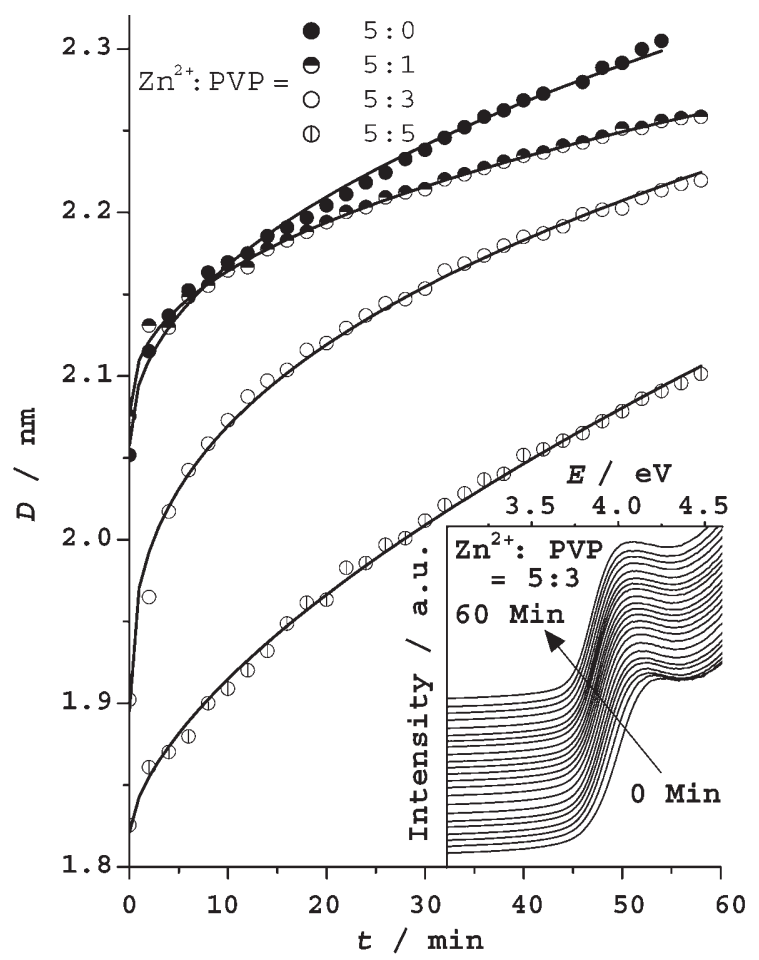

Figure 3. Variation in the size of $\mathrm{ZnO}$ nanocrystals shown as a function of time for different ratios of $\mathrm{Zn}^{2+}$ to $\mathrm{PVP}$ at a fixed concentration $(0.5 \mathrm{mmol})$ of $\mathrm{NaOH}$. The inset shows the UV-absorption curves for a typical system as a function of time ranging from 0-60 min.

tematic and substantial shift of the absorption edge to lower energies with increasing time, signifying a growth in the average particle size. The variations in average diameter, $D$, as a function of time, $t$, for the different ratios of $\mathrm{Zn}^{2+}$ to PVP, but with the same $\mathrm{NaOH}$ concentration, are shown in the main panel of Figure 3. In each case, there is a rapid and sustained growth of the nanocrystals with time, in spite of the presence of the capping agent. Prompted by the theory of Ostwald ripening, ${ }^{[11]}$ we tried to fit these curves to the expression $D^{x}-D_{0}{ }^{x}=k t$, in which $x=3$ in the case of Ostwald ripening. However, we found that it was impossible to fit any of the curves to the quoted formula, even by varying the value of $x$. This suggests that the growth process observed here is qualitatively different from the Ostwald ripening mechanism. This is not surprising in view of the presence of PVP as a capping agent that interferes with the usual ripening process; the ripening process being driven only by the gradient in surface energy. The mechanism of growth inhibition in the presence of a capping agent is not well understood and is beyond the scope of the Lifshitz Slyozov Wagner (LSW) theory ${ }^{[11]}$ leading to the Ostwald ripening. We find that an empirical fit in terms of $\left(D-D_{0}\right)^{x}=k t$ describes well the experimental results, as shown by the best fit results with the solid lines through the data points in Figure 3. The various values of $D_{0}, x$, and $k$ are listed in Table 1. This empirical growth equation suggests that the rate of growth over longer time periods is dependent on $x$. Thus, the largest value of $x$ for a $\mathrm{Zn}^{2+}$ :PVP ratio of 5:3 sug-
Table 1. $D_{0}, x$, and $k$ values for different ratios of $\mathrm{Zn}^{2+}$ to PVP.

\begin{tabular}{cccc}
\hline $\mathrm{Zn}^{2+}: \mathrm{PVP}$ & $D_{0}[\mathrm{~nm}]$ & $x$ & $k\left[\mathrm{~nm} \mathrm{~s}^{-1}\right]$ \\
\hline $5: 0$ & 2.05 & 2.15 & 0.038 \\
$5: 1$ & 2.07 & 2.38 & 0.033 \\
$5: 3$ & 1.89 & 2.76 & 0.070 \\
$5: 5$ & 1.82 & 1.58 & 0.022 \\
\hline
\end{tabular}

gests that this condition favors the eventual formation of smaller particles. This conclusion is supported by reports ${ }^{[2]}$ in which the specific ratio of $5: 3$ for $\mathrm{Zn}^{2+}$ :PVP concentrations was found to give rise to the smallest-sized particles after a sufficiently long reaction time.

A typical set of UV-absorption spectra at various concentrations of $\mathrm{NaOH}$ with a fixed ratio of $\mathrm{Zn}^{2+}$ to PVP (5:3) and after a reaction time of $160 \mathrm{~min}$ is given in the Supporting Information. The absorption edge appears clearly at different energies, varying non-monotonically and thereby indicating the complex dependence of the average nanocrystal size on $\mathrm{NaOH}$ concentration. More interestingly, the sharpness of the absorption edge is found to vary substantially as $\mathrm{NaOH}$ concentration changes. The most interesting dependence of growth on $\mathrm{NaOH}$ concentration was suggested by distinct non-monotonic variations in the UV-absorption spectra of the reaction mixture after a fixed time. We conducted transmission electron microscopy (TEM) and scanning tunneling microscopy (STM) to study the shape of compounds corresponding to different points shown in Figure 4. These data clearly show the presence of predominantly spherical particles of consistent sizes, confirming that the complex dependence on $\mathrm{NaOH}$ concentration is not due to any variation in shape of the nanocrystals. We then analyzed the absorption spectra obtained as a function of $\mathrm{NaOH}$ concentration at three different reaction times $(0$, 100, and $160 \mathrm{~min}$ ) and for different ratios of $\mathrm{Zn}^{2+}$ to PVP to obtain the average nanocrystal size and the FWHM of the size-distribution function, as described before. The results for $D_{\text {av }}$ are shown in the main panels of Figure 4. The relative percentage distribution, defined by $100(\Delta D / D)$, is similar for different reaction times, and hence is shown for only the $160 \mathrm{~min}$ reaction time in the corresponding insets. Figure $4 \mathrm{a}$ shows the expected result that the average particle size is largest in the absence of PVP $\left(\mathrm{Zn}^{2+}\right.$ to PVP ratio= $5: 0)$. A low $\mathrm{Zn}^{2+}$ to PVP ratio of $5: 1$ is also found to be inefficient in passivating the nanocrystals (Figure 4b), and we observe minimal changes compared to the uncapped system (Figure 4a) in terms of both size and size distribution. At a few concentrations, we even find slightly larger nanocrystals in the case of the 5:1 ratio than in the absence of PVP. However, we do not consider these slight changes to be significant for the following reasons: It is known that even a slight change in the water content may influence the size significantly. A small change in the water content, below the controllable limit, may be the reason for the slight increase observed for the 5:1 case. However, the average size of the nanocrystal decreases systematically as the PVP concentration increases, as shown in Figure $4 c$ and d. Interestingly, 
a) $5: 0$

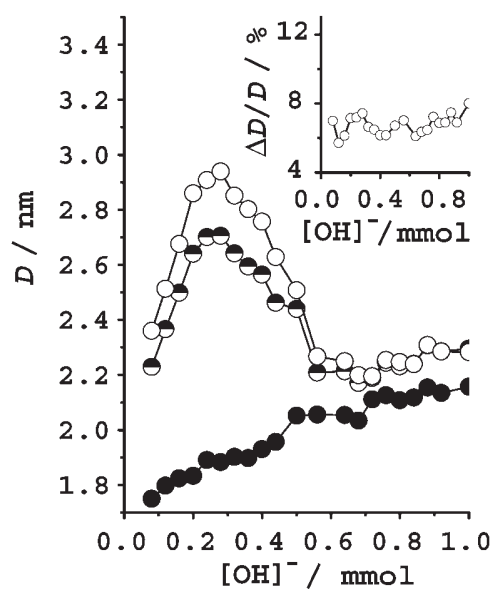

c) $5: 3$

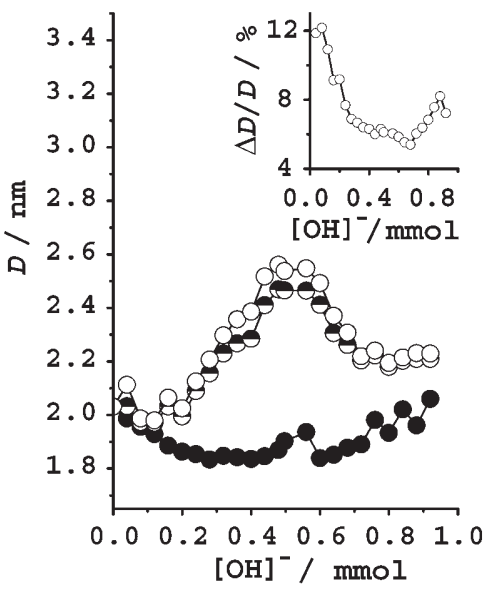

b) $5: 1$

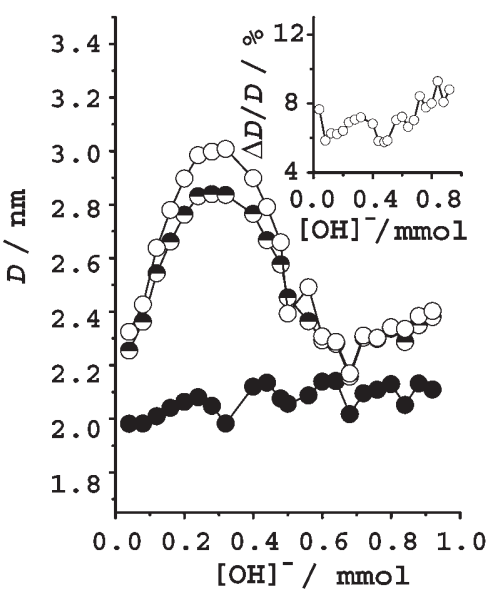

d) $5: 5$

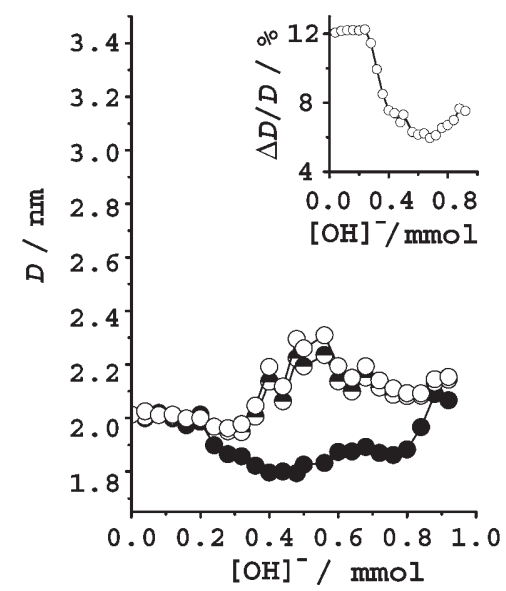

Figure 4. Variation in the size of $\mathrm{ZnO}$ nanocrystals plotted as a function of $\mathrm{NaOH}$ concentration at times 0 min (closed circles), 100 min (half-open circles), and 160 min (open circles) for $\mathrm{Zn}^{2+}$ to PVP ratios of (a) $5: 0$, (b) $5: 1$, (c) 5:3, and (d) 5:5. The corresponding insets show the variation in size distribution at $160 \mathrm{~min}$.

Figure $4 \mathrm{c}$ and $\mathrm{d}$ suggest the formation of $\mathrm{ZnO}$ nanocrystals prior to the addition of $\mathrm{NaOH}$ (the zero of the $x$-axis). This is not observed in the absence of PVP (Figure 4a), nor for small amounts of PVP (Figure 4b). These preformed $\mathrm{ZnO}$ nanocrystals can be explained on the basis of our observation that the large concentration of PVP makes the solution basic; this produces enough hydroxyl ions to produce detectable quantities of $\mathrm{ZnO}$-like nanocrystals even prior to the addition of any $\mathrm{NaOH}$.

The results shown in Figure 4 reveal that the dependencies of $D_{\text {av }}$ on the concentration of $\mathrm{NaOH}$ for all concentrations of PVP are essentially similar. At the start of the reaction, that is, at $t=0$, represented by closed circles in Figure 4, the average size is not particularly sensitive to the concentration of $\mathrm{NaOH}$, though the lower concentrations of PVP show a slight monotonic increase as the $[\mathrm{OH}]^{-}$concentration increases (panels a and b). At higher concentrations of PVP (panels $\mathrm{c}$ and d), the average size shows an initial slight decrease, forming a shallow broad minimum as the concentration of $\mathrm{NaOH}$ increases. Interestingly, a lower con- centration of PVP (panels a and b) allows a systematic growth of the nanocrystal size over time, even at the smallest $\mathrm{NaOH}$ concentration, whereas at higher PVP concentrations (panels $\mathrm{c}$ and $\mathrm{d}$ ), the $\mathrm{ZnO}$ nanocrystals exhibit hardly any growth below a critical $\mathrm{NaOH}$ concentration, as evidenced by the overlap of the three curves for the three different times in the lower $\mathrm{NaOH}$ concentration range. In fact, the concentration of $\mathrm{NaOH}$ required before growth of the nanocrystals can be observed increases as the PVP concentration increases. This suggests that in the presence of PVP, even before the addition of $\mathrm{NaOH}$, the clusters of $\mathrm{ZnO}$ are well passivated, thereby inhibiting any further growth of the nanocrystals.

After the reaction has progressed for some time $(t=100$ or $160 \mathrm{~min}$ ), the average size of the nanocrystals becomes a non-monotonic function of the $\mathrm{NaOH}$ concentration, reaching a distinct maximum for an intermediate concentration of $\mathrm{NaOH}$ and finally a low saturation value near the highest concentration investigated here. The initial increase in size is expected, due to the increase in the number of hydroxyl ions available for the reaction. However, in each case, the size of the nanocrystals becomes progressively smaller as $\mathrm{NaOH}$ concentration increases beyond a certain concentration. To investigate this observation, we first note that the progress of the reaction proceeds by dehydration of $\mathrm{Zn}(\mathrm{OH})_{2}$ to produce $\mathrm{ZnO}$ nanocrystal and water. It is well known that the presence of even a small amount of water increases significantly the rate of growth of $\mathrm{ZnO}$ nanocrystals. ${ }^{[6,12]}$ In the presence of excess $\mathrm{NaOH}$, the unreacted $\mathrm{NaOH}$ acts as a scavenger of water, absorbing moisture from the solution and thus maintaining relatively anhydrous conditions. This explains the observation of smaller-sized nanocrystals at a higher concentration of $\mathrm{NaOH}$ beyond a certain threshold concentration. We proved this hypothesis by carrying out the reaction in the presence of anhydrous silica gel for different concentrations of $\mathrm{NaOH}$; this was found to slow down the growth of the nanocrystal size. In particular, for the $\mathrm{NaOH}$ concentration corresponding to the maximum nanocrystal sizes shown in Figure 4, we observed a drastic reduction in the nanocrystal 
size in the presence of silica gel, consistent with the extent of decrease in size in the presence of excess $\mathrm{NaOH}$ shown in Figure 4. Typical results of the experiment with silica gel are available in the Supporting Information.

The insets to Figure 4a and $\mathrm{b}$ reveal a slight monotonic increase in the relative distribution at low PVP concentration as $\mathrm{NaOH}$ concentration increases. Interestingly, at higher concentrations of PVP (insets to Figure $4 \mathrm{c}$ and d), we observe a striking decrease in the size distribution or a narrowing effect. As discussed earlier, in the presence of high concentrations of PVP and in the absence or at a low concentration of $\mathrm{NaOH}$, the $\mathrm{ZnO}$-like clusters are formed from the oxide ions of the PVP and isopropanol in the medium, catalyzed by the slight basicity of the medium. In this case, the rate-determining step of the reaction is the formation of the $\mathrm{ZnO}$-like clusters, and these clusters are immediately capped efficiently with PVP. Therefore, further ripening leading to more uniformly sized clusters is strongly suppressed at higher PVP concentrations by the effective passivation. This is also suggested by the fact that there is hardly any growth of the nanocrystal size with time, indicated by the overlap of the three time $(0,100$, and $160 \mathrm{~min})$ plots, for the large PVP concentrations (Figure $4 \mathrm{c}$ and d) in the low $\mathrm{NaOH}$ concentration range. Hence, we observe a broad size distribution in such cases. Beyond a certain critical concentration of $\mathrm{NaOH}$, approximately $0.1-0.2 \mathrm{mmol}$ in Figure $4 \mathrm{c}$ and $\mathrm{d}$, respectively, there is clear growth of the nanocrystal size with time. This suggests that at these higher concentrations, $\mathrm{NaOH}$ is effective in reacting with the small, preformed $\mathrm{ZnO}$ clusters in spite of the presence of PVP. This thereby leads to growth, though at a much slower rate than in the uncapped case. Removal of the capping agent at higher concentrations of $\mathrm{NaOH}$ also allows for a ripening process to compete with the passivating process by PVP, and this leads to a higher degree of uniformity in size, as observed here. Notably, the minimum in the relative distribution corresponds to the same $\mathrm{NaOH}$ concentration that is required to reach the low size range in the higher $\mathrm{NaOH}$ concentration range. If the $\mathrm{NaOH}$ concentration is higher than this optimal value, the relative distribution becomes larger, leading to a poorer quality product, though the average particle size remains approximately the same. On the other hand, a lower concentration of $\mathrm{NaOH}$ gives rise to a larger average size for the nanocrystals. Thus, the present study establishes the necessity to use the optimal $\mathrm{NaOH}$ concentration to obtain small nanocrystals with a minimum size distribution.

\section{Conclusion}

We have presented a simple approach to obtain accurate estimates of size-distribution functions in nanocrystal systems from UV-absorption spectra. General validity of this method has been shown by applying it to diverse semiconductor nanocrystals of various sizes. The unique ability of this novel approach to provide a detailed understanding of the complex growth and a route to rational synthesis of the high-quality nanocrystal is illustrated by the case of $\mathrm{ZnO}$ nanocrystal growth with PVP as the capping agent. The complexity of the process is revealed at a microscopic level by its qualitative differences to the Ostwald ripening mechanism, and at a macroscopic level in terms of non-monotonic dependencies of the size and the size-distribution function on different reaction parameters. Additionally, the optimal conditions for $\mathrm{ZnO}$ nanocrystal synthesis with the narrowest size distribution are defined, paving the way for a rational synthesis.

\section{Experimental Section}

A typical synthesis of the $\mathrm{ZnO}$ nanocrystals involved dissolving $0.25 \mathrm{mmol}$ of zinc acetate in $25 \mathrm{~mL}$ of isopropanol under constant stirring at $50^{\circ} \mathrm{C}$. Various specific amounts of PVP were added to this solution and stirring was continued. These solutions were hydrolyzed by the addition of various concentrations of $\mathrm{NaOH}$ in isopropanol, and $\mathrm{UV}$-absorption spectra of these solutions were measured at various times by using a Perkin-Elmer double-beam LM-35 spectrophotometer. Pure $i \mathrm{PrOH}$ was used as a reference blank solution for the absorption experiments. The synthesis and characterization of the other compounds shown in Figure 1 are reported in the literature. ${ }^{[4,8,9]}$

To extract the size distribution from an analysis of the UV-absorption edge, we first note that any property of a collection of non-correlated particles is simply the average of the properties of all of the individual particles. Specifically, the UV-absorption spectrum of any nanocrystal sample is obtained by the superimposition of the individual UV-absorption spectra representing each specific size of nanocrystal weighted by its relative abundance. Thus, by using the UV-absorption spectrum of the bulk as the reference spectral shape for each contribution, and the energy shift of the absorption edge for any given size from the literature, ${ }^{[4,13-15]}$ the expected spectrum can be easily simulated for any given size distribution. We assume that the size dispersion follows a statistically determined Gaussian distribution, characterized by the peak position, $D_{\text {av }}$, which corresponds to the average diameter of the nanocrystals and the full width at half maximum (FWHM), and $\Delta D$, which quantifies the size dispersion. We then systematically vary the parameters $D_{\text {av }}$ and $\Delta D$ to minimize the difference between the simulated spectrum and the experimental spectrum within the leastsquared-error approach, thus determining the size-distribution function most consistent with the experimentally observed absorption spectrum.

\section{Acknowledgements}

This work was supported by the Department of Science and Technology, Government of India. 
[1] a) Thin film solar cells (Eds.: K. L. Chopra, S. R. Das), Plenum, New York, 1983; b) S. Hingorani, V. Pillai, P. Kumar, M. S. Multani, D. O. Shah, Mater. Res. Bull. 1993, 28, 1303.

[2] L. Guo, S. Yang, C. Yang, P. Yu, J. Wang, W. Ge, G. K. L. Wong, Chem. Mater. 2000, 12, 2268.

[3] N. S. Pesika, Z. Hu, K. J. Stebe, P. C. Searson, J. Phys. Chem. B 2002, 106, 6985.

[4] R. Viswanatha, S. Sapra, B. Satpati, P. V. Satyam, B. N. Dev, D. D. Sarma, J. Mater. Chem. 2004, 14, 661.

[5] P. V. Radovanovic, N. S. Norberg, K. E. McNally, D. R. Gamelin, J. Am. Chem. Soc. 2002, 124, 15192.

[6] R. Viswanatha, S. Sapra, S. Sen Gupta, B. Satpati, P. V. Satyam, B. N. Dev, D. D. Sarma, J. Phys. Chem. B 2004, 108, 6303.

[7] M. Zinke-Allmang, L. C. Feldman, S. Nakahara, Appl. Phys. Lett. 1987, 51,975
[8] J. Nanda, S. Sapra, D. D. Sarma, N. Chandrasekharan, G. Hodes, Chem. Mater. 2000, 12, 1018.

[9] B. O. Dabbousi, C. B. Murray, M. F. Rubner, M. G. Bawendi, Chem. Mater. 1994, 6, 216.

[10] X. Peng, J. Wickham, A. P. Alivisatos, J. Am. Chem. Soc. 1998, 120, 5343.

[11] a) I. M. Lifshitz, V. V. Slyozov, Phys. Chem. Solids 1961, 19, 35; b) C. Wagner, Z. Elektrochem. 1961, 65, 581.

[12] E. A. Meulenkamp, J. Phys. Chem. B 1998, 102, 5566.

[13] S. Sapra, N. Shanthi, D. D. Sarma, Phys. Rev. B 2002, 66, 205202.

[14] S. Sapra, D. D. Sarma, Phys. Rev. B 2004, 69, 125304.

[15] R. Viswanatha, S. Sapra, T. Saha-Dasgupta, D. D. Sarma, Phys. Rev. $B$ 2005, 72, 045333 .

Received: June 4, 2005 Published online: October 17, 2005 